\title{
Cosmic String Zero Modes and Multiple Phase Transitions
}

\author{
Stephen C. Davis ${ }^{1 *}$, Anne-Christine Davis ${ }^{1 \dagger}$ and Warren B. Perkins ${ }^{2 \ddagger}$ \\ ${ }^{1}$ Department of Applied Mathematics and Theoretical Physics, \\ University of Cambridge, Cambridge, CB3 9EW, UK. \\ ${ }^{2}$ Department of Physics, University of Wales Swansea, \\ Singleton Park, Swansea, SA2 8PP, Wales
}

July 15, 2021

\begin{abstract}
The zero modes and current carrying capability of a cosmic string formed at one phase transition can be modified at subsequent phase transitions. A new, generalised index theorem is derived that is applicable to theories with multiple phase transitions. This enables us to investigate the fate of string zero modes during sequences of phase transitions in a variety of models. Depending on the couplings that the breaking introduces, the zero modes may be destroyed and the superconductivity of the string removed, and thus vortons dissipate. We discuss the features of the theory that are required to produce this behaviour and consider the implications of spectral flow.
\end{abstract}

DAMTP/97-39

SWAT $/ 140$

\footnotetext{
*S.C.Davis@damtp.cam.ac.uk

${ }^{\dagger}$ A.C.Davis@damtp.cam.ac.uk

${ }^{\ddagger}$ w.perkins@swansea.ac.uk
} 


\section{Introduction}

Topological defects, and in particular cosmic strings, arise in many grand unified theories [1]. They are likely to be copiously produced during phase transitions in the early universe. A network of cosmic strings could explain the observed anisotropy in the microwave background radiation and the large scale structure of the Universe [1]. In the past few years it has been realised that they may have considerably richer microstructure than previously realised [2]. In particular, the presence of fermion zero modes [3] in the spectrum of a cosmic string has profound implications for the cosmology of the defects. Zero modes render the string superconducting 伆, allowing currents of up to $10^{20}$ Amps to flow along the string. Stable relics, vortons, can form as collapsing string loops are stabilised by the angular momentum of the trapped charge carriers [5]. These relics can overclose the Universe unless stringent constraints are applied to the string model [6].

The cosmological implications of the vortons are most pronounced when the Universe has become matter dominated. If they decay during the era of radiation domination the cosmological catastrophe may be avoided and the vorton bounds evaded. However, it has recently been realised that subsequent phase transitions can have a considerable effect on the microphysics of cosmic strings. Zero modes on cosmic strings can be both created [7] and destroyed [8], thus creating or destroying currents on strings. In this paper we discuss the fate of vortons and string superconductivity as the strings encounter subsequent phase transitions in a systematic fashion.

In section 2 we derive an index theorem giving the number of zero modes for a general mass matrix. Whilst index theorems have been derived before [9, 10] they have been more restrictive in their validity and have only been able to determine the difference in right-moving and left-moving zero modes. Our index theorem has much more general applicability and can give a bound on the number of zero modes.

In section 3 we discuss the fate of zero modes on strings formed at the breaking of an $\mathrm{SO}(10)$ Grand Unified symmetry [8]. At the electroweak phase transition these zero modes acquire a small mass which leads to dissipation of the string current. This allows vortons to decay and weakens the cosmological bounds on such models 11.

In other models the zero modes survive subsequent transitions allowing the associated vortons to persist. Such behaviour is displayed by a toy model

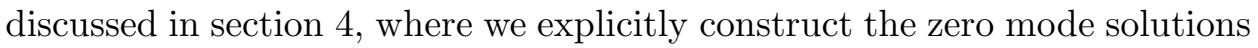
of the Dirac equation after the symmetry breaking. 
We compare these two models in section 5 and consider the implications of spectral flow. An important feature that allows zero modes to be removed is the presence of a particle that mixes with the zero mode after the transition. The implications of such couplings for current build up before the transition are also considered. Finally, we summarise our conclusions.

\section{Zero Mode Index}

Cosmic strings form in models with vacuum manifolds which are not simply connected. For example in a $U(1)$ model, with potential $\left(|\phi|^{2}-\eta^{2}\right)^{2}$, stable solutions exist with $\phi=\eta e^{i \theta}$ at $r=\infty$. In order for the total energy to be finite, a non-zero gauge field is needed to give a vanishing covariant derivative at $r=\infty$. In a more general theory, involving a larger group, $G$, string solutions take the form

$$
\phi(r, \theta)=e^{i T_{s} \theta} \phi(r), A_{\theta}=\frac{1}{e r} T(r),
$$

where $T_{s}$ is a generator of $G$ that is broken by $\phi$. The choice of $T_{s}$ is restricted by the fact that $\phi$ must be single valued. $\phi(r)$ is equal to the usual VEV of $\phi$ at $r=\infty$, and must be regular at $r=0$. $T(r)$ obeys $T(0)=0, T(\infty)=T_{s}$.

In a general theory, $T_{s}$ will affect different components of $\phi$ differently. This means that the various parts of $\phi$ can have a wide range of winding numbers. In a theory with multiple phase transitions, the additional Higgs fields will be affected in the same way. It may also be necessary to alter $T_{s}$ at phase transitions to make the new Higgs fields single valued.

In a theory with $n_{\mathrm{f}}$ two-component fermions, the fermionic part of the Lagrangian is

$$
\mathcal{L}_{\mathrm{f}}=\bar{\psi}_{\alpha} i \sigma^{\mu} D_{\mu} \psi_{\alpha}-\frac{1}{2} i \bar{\psi}_{\alpha} M_{\alpha \beta} \psi_{\beta}^{c}+\text { (h.c.) }
$$

where $\psi_{\beta}^{c}=i \sigma^{2} \bar{\psi}_{\beta}^{T}$. If $M_{\alpha \beta}$ depends on $\theta$, as would be expected if $M_{\alpha \beta}$ arose from the Higgs field of the string, then it is possible that the field equations will have non-trivial zero energy solutions. Solutions with only $r$ and $\theta$ dependence can be split up into eigenstates of $\sigma^{3}: \psi_{\alpha}^{L}, \psi_{\alpha}^{R}$. Such solutions have zero energy. If we solve the equations of motion in the background of a cosmic string, the field equations become

$$
e^{i \theta}\left(\partial_{r}+\frac{i}{r} \partial_{\theta}+e A_{\theta}\right) \psi_{\alpha}^{L}+M_{\alpha \beta} \psi_{\beta}^{L *}=0
$$




$$
e^{-i \theta}\left(\partial_{r}-\frac{i}{r} \partial_{\theta}-e A_{\theta}\right) \psi_{\alpha}^{R}-M_{\alpha \beta} \psi_{\beta}^{R *}=0,
$$

where $A_{\theta}$ is the string gauge field. If $z$ and $t$ dependence is added to the solutions they will correspond to currents flowing along the string. Their direction is left for those corresponding to (3), and right for (伍). In order to be physically relevant the solutions must be normalisable. Let $N_{L}$ and $N_{R}$ be the number of such solutions to (3) and (4) respectively. We attempt to derive an expression for them by generalising the analysis in ref. [3], which involves removing the $\theta$ dependence of the problem, and then considering solutions near $r=\infty$ and $r=0$.

Choose the $\psi_{\alpha}$ s to be eigenstates of the string gauge field, with eigenvalues $q_{\alpha}$. The $q_{\alpha}$ will depend on the fermion charges and the winding numbers of the various components of the Higgs fields. Since the mass terms in (2) are gauge invariant, the angular dependence of the mass matrix must be

$$
M_{\alpha \beta}(r, \theta)=C_{\alpha \beta}(r) e^{i\left(q_{\alpha}+q_{\beta}\right) \theta} \quad \text { (no summation) . }
$$

The $\theta$ dependence can also be factored out of the $\psi_{\alpha}$ s.

$$
\begin{aligned}
\psi_{\alpha}^{L} & =e^{i\left(q_{\alpha}-\frac{1}{2}\right)}\left(U_{\alpha}^{L}(r) e^{i l \theta}+V_{\alpha}^{L *}(r) e^{-i l \theta}\right), \\
\psi_{\alpha}^{R} & =e^{i\left(q_{\alpha}+\frac{1}{2}\right)}\left(U_{\alpha}^{R}(r) e^{i l \theta}+V_{\alpha}^{R *}(r) e^{-i l \theta}\right) .
\end{aligned}
$$

First consider left moving zero modes. Putting (5,7) into (3) gives equations for $U_{\alpha}^{L}$ and $V_{\alpha}^{L}$. As $r \rightarrow \infty, C_{\alpha \beta}=O(1)$ and $e A_{\theta}=O(1 / r)$, so

$$
\begin{gathered}
\partial_{r} U_{\alpha}^{L}+C_{\alpha \beta}(\infty) V_{\beta}^{L}=0, \\
\partial_{r} V_{\alpha}^{L}+C_{\alpha \beta}(\infty) U_{\beta}^{L}=0 .
\end{gathered}
$$

Diagonalising $C_{\alpha \beta}$ gives $2 n_{\mathrm{f}}$ complex solutions proportional to $\exp \left( \pm \lambda_{\alpha}\right)$, where $\lambda_{\alpha}$ are $C_{\alpha \beta}$ 's eigenvalues. Thus, assuming all $\lambda_{\alpha} \neq 0$, exactly $n_{\mathrm{f}}$ of these large $r$ solutions are normalisable at $r=\infty$.

The Higgs fields, and hence $M_{\alpha \beta}$, are regular at the origin, so as $r \rightarrow 0$, $C_{\alpha \beta}=O(1)$, and $e A_{\theta}=O(r)$. Thus

$$
\begin{aligned}
& \left(\partial_{r}-\frac{q_{\alpha}-\frac{1}{2}+l}{r}\right) U_{\alpha}^{L}+C_{\alpha \beta} V_{\beta}^{L *}=0, \\
& \left(\partial_{r}-\frac{q_{\alpha}-\frac{1}{2}-l}{r}\right) V_{\alpha}^{L}+C_{\alpha \beta} U_{\beta}^{L *}=0 .
\end{aligned}
$$


To leading order, the small $r$ solutions are

$$
\begin{aligned}
U_{\alpha}^{L} & \sim r^{q_{\alpha}-\frac{1}{2}+l}, \\
V_{\beta}^{L} & \sim O(1) r^{q_{\beta}+\frac{1}{2}+l} \quad \forall \beta, \\
U_{\beta}^{L} & \sim O(1) r^{q_{\beta}+\frac{3}{2}+l} \quad \forall \beta \neq \alpha,
\end{aligned}
$$

where each choice of $\alpha=1 \ldots n_{\mathrm{f}}$ gives one complex solution, and

$$
\begin{aligned}
V_{\alpha}^{L} & \sim r^{q_{\alpha}-\frac{1}{2}-l} \\
U_{\beta}^{L} & \sim O(1) r^{q_{\beta}+\frac{1}{2}-l} \quad \forall \beta, \\
V_{\beta}^{L} & \sim O(1) r^{q_{\beta}+\frac{3}{2}-l} \quad \forall \beta \neq \alpha,
\end{aligned}
$$

which gives a total of $2 n_{\mathrm{f}}$ independent complex solutions. For given $l$ and $\alpha$, (12) will be normalisable (for small $r$ ) if $l \geq-q_{\alpha}+1 / 2$. If $l \leq q_{\alpha}-1 / 2$ then (13) will be normalisable. Thus for a given $l$ the number of well behaved small $r$ solutions is

$$
N_{L}^{0}(l)=\sum_{\alpha=1}^{n_{\mathrm{f}}} \mathrm{I}\left[l \leq q_{\alpha}-\frac{1}{2}\right]+\mathrm{I}\left[l \geq-q_{\alpha}+\frac{1}{2}\right]
$$

where $\mathrm{I}[X]$ equals 1 if $X$ is true, and 0 if $X$ is false. What we are actually interested in is the number of solutions that are normalisable for all $r$ $\left(N_{L}(l)\right)$. Each such solution will be equal to some combination of the $n_{\mathrm{f}}$ well behaved solutions to (8,9) at large $r$, and a combination of the $N_{L}^{0}(l)$ suitable solutions to (10,11) for small $r$. If there are only $n_{\mathrm{f}}$, or less, suitable small $r$ solutions, then in general any combination of the large $r$ solutions will not be well behaved at $r=0$. If there are $n_{\mathrm{f}}+k$ suitable small $r$ solutions, then $k$ independent combinations of the large $r$ solutions will be well behaved everywhere. It may be possible to get more solutions by fine tuning the theory, in which case the index derived would be a lower bound.

The number of normalisable solutions for a given $l$ is

$$
N_{L}(l)=\left[N_{L}^{0}(l)-n_{\mathrm{f}}\right]_{+},
$$

where $[x]_{+}$is defined to be equal to zero if $x<0$, and $x$ if $x \geq 0$.

This is not true if the equations obtained from (3) can be split into several independent sets. This will occur when $M_{\alpha \beta}$ is a direct product of mass matrices. In this case the mass matrix can be split up into smaller matrices, which can be analysed individually. Even when $M_{\alpha \beta}$ is not a direct 
product of other matrices, it may still be possible to split the equations into two independent sets. This case will be considered separately later.

Since $U_{\alpha}^{L}$ and $V_{\alpha}^{L}$ are determined by real equations, each complex solution gives two real solutions. This suggests that the total number of left moving zero modes, $N_{L}$, is $2 \sum_{l} N_{L}(l)$. However, as can be seen from (7), solutions for $l=k$ and $l=-k$ are equal. For $l=0 U_{\alpha}^{L}= \pm V_{\alpha}^{L}$, so one of $\psi_{\alpha}^{L}$ 's solutions is zero. Thus the total number of independent real solutions is

$$
N_{L}=\sum_{l} N_{L}(l)=\sum_{l}\left[\sum_{\alpha=1}^{n_{\mathrm{f}}}\left(\mathrm{I}\left[l \leq q_{\alpha}-\frac{1}{2}\right]+\mathrm{I}\left[l \geq-q_{\alpha}+\frac{1}{2}\right]\right)-n_{\mathrm{f}}\right]_{+} .
$$

The summation is over all values of $l$ that give single valued $\psi$. Since all the Higgs fields which make up $M_{\alpha \beta}$ are single valued, (5) implies all $q_{\alpha}$ or all $q_{\alpha}-1 / 2$ are integers (assuming $M_{\alpha \beta}$ is not a product of smaller matrices), in which case respectively $l-1 / 2$ or $l$ is an integer.

A similar analysis can be applied to right moving zero modes. For large $r$ the behaviour is the same. For small $r$, solutions are well behaved if $l \geq q_{\alpha}+1 / 2$ or $l \leq-q_{\alpha}-1 / 2$. This gives

$$
N_{R}=\sum_{l} N_{R}(l)=\sum_{l}\left[\sum_{\alpha=1}^{n_{\mathrm{f}}}\left(\mathrm{I}\left[l \leq-q_{\alpha}-\frac{1}{2}\right]+\mathrm{I}\left[l \geq q_{\alpha}+\frac{1}{2}\right]\right)-n_{\mathrm{f}}\right]_{+} .
$$

If $q_{\alpha}$ is positive then one or two of the I [...] terms will be non-zero. If $q_{\alpha}$ is negative, one or zero of them will be non-zero. By splitting $q_{\alpha}$ into positive and negative eigenvalues, and ordering them, (16) and (17) can be simplified. If there are $n_{+}$positive and $n_{-}$negative $q_{\alpha}$ s then, after reordering, $q_{\alpha}=$ $\left(p_{1}, p_{2} \ldots p_{n_{+}},-g_{1},-g_{2} \ldots-g_{n_{-}}, 0 \ldots 0\right)$, where $p_{j}, g_{j}>0$. Clearly if the string gauge eigenvalues are not integer, there are no zeros. The I [...] terms in (16) and (17) can be combined to give

$$
\begin{aligned}
& N_{L}=\sum_{l}\left[\sum_{j=1}^{n_{+}} \mathrm{I}\left[-p_{j}+\frac{1}{2} \leq l \leq p_{j}-\frac{1}{2}\right]-\sum_{j=1}^{n_{-}} \mathrm{I}\left[-g_{j}-\frac{1}{2}<l<g_{j}+\frac{1}{2}\right]\right]_{+}, \\
& N_{R}=\sum_{l}\left[\sum_{j=1}^{n_{-}} \mathrm{I}\left[-g_{j}+\frac{1}{2} \leq l \leq g_{j}-\frac{1}{2}\right]-\sum_{j=1}^{n_{+}} \mathrm{I}\left[-p_{j}-\frac{1}{2}<l<p_{j}+\frac{1}{2}\right]\right]_{+} .
\end{aligned}
$$


These expressions can be further simplified if the eigenvalues are ordered. If $p_{1} \geq p_{2} \geq \ldots \geq p_{n_{+}}>0$ and $g_{1} \geq g_{2} \geq \ldots \geq g_{n_{-}}>0$, it is possible to evaluate the $l$ summation by considering cancellation of the $p_{j}$ and $g_{j}$ terms. This gives

$$
\begin{aligned}
& N_{L}=\sum_{j=1}^{\min \left(n_{-}, n_{+}\right)} 2\left[p_{j}-g_{j}\right]_{+}+\sum_{j=n_{-}+1}^{n_{+}} 2 p_{j}, \\
& N_{R}=\sum_{j=1}^{\min \left(n_{-}, n_{+}\right)} 2\left[g_{j}-p_{j}\right]_{+}+\sum_{j=n_{+}+1}^{n_{-}} 2 g_{j} .
\end{aligned}
$$

Taking the difference of these results gives

$$
\begin{aligned}
\mathcal{I}_{\Delta}=N_{L}-N_{R} & =\sum_{j=1}^{\min \left(n_{-}, n_{+}\right)} 2\left(p_{j}-g_{j}\right)+\sum_{j=n_{-}+1}^{n_{+}} 2 p_{j}-\sum_{j=n_{+}+1}^{n_{-}} 2 g_{j}(22) \\
& =\sum_{\alpha=1}^{n_{\mathrm{f}}} 2 q_{\alpha}=\frac{1}{2 \pi i}[\ln \operatorname{det} M]_{\theta=0}^{2 \pi} .
\end{aligned}
$$

This is in agreement with another index theorem obtained elsewhere [9, 10]. The other index theorem was obtained by a different method and only gave $\mathcal{I}_{\Delta}$, not $N_{L}$ and $N_{R}$.

The total number of zero modes is

$$
\mathcal{I}=N_{L}+N_{R}=\sum_{j=1}^{\min \left(n_{-}, n_{+}\right)} 2\left|p_{j}-g_{j}\right|+\sum_{j=n_{-}+1}^{n_{+}} 2 p_{j}+\sum_{j=n_{+}+1}^{n_{-}} 2 g_{j},
$$

where only one of the last 2 terms contributes, depending on whether $n_{+}$or $n_{-}$is bigger. This is also true of (22) and (20,21).

If $\mathcal{I}$ is to be zero, then for every positive $q_{\alpha}$ there must be one negative $q_{\beta}$ with the same magnitude. If every fermion field couples to a Higgs field with winding number zero, this will be the case.

The above approach fails if $C_{\alpha \beta}$ is of the form

$$
\left(\begin{array}{cc}
0 & J_{\alpha \beta} \\
K_{\alpha \beta} & 0
\end{array}\right)
$$

As $C_{\alpha \beta}$ is assumed to have no zero eigenvalues, $J_{\alpha \beta}$ and $K_{\alpha \beta}$ are both $n \times n$ matrices, where $n=n_{\mathrm{f}} / 2$. In this case when (5, (7) are substituted into (3), 
two independent sets of equations are obtained. Expressions for $N_{L}$ and $N_{R}$ can found by considering just one set of these solutions. Putting

$$
\psi_{\alpha}^{L}=e^{i\left(q_{\alpha}-\frac{1}{2}\right)} \begin{cases}U_{\alpha}^{L}(r) e^{i l \theta} & \alpha=1 \ldots n \\ \left.V_{\alpha}^{L *}(r) e^{-i l \theta}\right) & \alpha=n+1 \ldots n_{\mathrm{f}}\end{cases}
$$

and (5) into (3) gives (8,9) for large $r$ and (10,11) for small $r$, but with a more restricted range on the indices. For large $r, n$ of the $n_{\mathrm{f}}$ complex solutions are normalisable. For a given $l$ at small $r$, there is one normalisable solution for each $q_{\alpha}$ satisfying $l \geq-q_{\alpha}+1 / 2(\alpha=1 \ldots n)$ or $l \leq q_{\alpha}-1 / 2$ $\left(\alpha=n+1 \ldots n_{\mathrm{f}}\right)$.

Matching the solutions for large and small $r$ gives

$$
N_{L}(l)=\left[\sum_{\alpha=1}^{n}\left(\mathrm{I}\left[l \leq q_{\alpha+n}-\frac{1}{2}\right]+\mathrm{I}\left[l \geq-q_{\alpha}+\frac{1}{2}\right]\right)-n\right]_{+} .
$$

The solutions for different $l$ are independent (unlike the previously considered cases), so the total number of real solutions $\left(N_{L}\right)$ is just twice the number of complex solutions, thus $N_{L}=2 \sum_{l} N_{L}(l)$. A similar expression can be obtained for $N_{R}$.

The I [...] terms in (27) can be combined. Defining $g_{j}=-q_{j}$ and $p_{j}=$ $q_{j+n}$ for $j=1 \ldots n$, (27) and the corresponding expression for $N_{R}$, become

$$
\begin{aligned}
& N_{L}=2 \sum_{l}\left[\sum_{j=1}^{n}\left(\mathrm{I}\left[g_{j}+\frac{1}{2} \leq l \leq p_{j}-\frac{1}{2}\right]-\mathrm{I}\left[p_{j}-\frac{1}{2}<l<g_{j}+\frac{1}{2}\right]\right)\right]_{+} \\
& N_{R}=2 \sum_{l}\left[\sum_{j=1}^{n}\left(\mathrm{I}\left[p_{j}+\frac{1}{2} \leq l \leq g_{j}-\frac{1}{2}\right]-\mathrm{I}\left[g_{j}-\frac{1}{2}<l<p_{j}+\frac{1}{2}\right]\right)\right]_{+}
\end{aligned}
$$

If the string gauge eigenvalues are reordered, so that $p_{1} \geq \ldots \geq p_{n}$ and $g_{1} \geq \ldots \geq g_{n}$, the expressions reduce to (20) and (21), with $n_{+}=n_{-}=n$. This is not identical to the previous result, since $p_{j}$ and $g_{j}$ are defined differently.

When there are just two fermion fields involved, all the results reduce to

$$
\begin{aligned}
\mathcal{I}_{\Delta} & =2\left(q_{1}+q_{2}\right), \\
\mathcal{I} & =\left|\mathcal{I}_{\Delta}\right| .
\end{aligned}
$$




\section{An $S O(10)$ GUT with Strings}

One example of a phenomologically credible grand unified theory (GUT) has the symmetry breaking

$$
\begin{array}{rll}
S O(10) & \stackrel{\Phi_{126}}{\longrightarrow} & S U(5) \times Z_{2} \\
& \stackrel{\Phi_{45}}{\longrightarrow} & S U(3)_{c} \times S U(2)_{L} \times U(1)_{Y} \times Z_{2} \\
& \stackrel{\Phi_{10}}{\longrightarrow} & S U(3)_{c} \times U(1)_{Q} \times Z_{2} .
\end{array}
$$

The discrete $Z_{2}$ symmetry allows the formation of topologically stable cosmic strings. One possibility is an abelian string, in which case

$$
\Phi_{126}=e^{i n \theta} \phi_{0} f(r),
$$

where $\phi_{0}$ is the usual VEV of $\Phi_{126}, f(0)=0$ and $f(\infty)=1$. To give a vanishing covariant derivative at infinity there is also a non-zero gauge field. The only stable abelian strings have $|n|=1$, but higher winding number strings may have long lifetimes. Nonabelian strings also form in this model, but they do not have zero modes at high temperatures, and so will be ignored [8].

The string gauge field has a non-trivial effect on the electroweak Higgs field, $\Phi_{10}$. Thus

$$
\Phi_{10}=\left[H_{0} e^{i m \theta}+H_{0}^{\prime} e^{-i m \theta}\right] h(r),
$$

where $H_{0}$ and $H_{0}^{\prime}$ are the usual VEVs of the components of $\Phi_{10}, h(\infty)=1$, and $h(0)$ is small $(m=0)$ or zero $(m \neq 0)$. There is also a non-vanishing electroweak gauge field. $m$ is determined by the GUT string, and is equal to the nearest integer to $-n / 5$, so the electroweak Higgs field does not wind around a topologically stable abelian string.

Of the three Higgs fields, only $\Phi_{126}$ and $\Phi_{10}$ couple to fermions. Only right-handed neutrinos couple to $\phi_{0}$, while neutrinos of either helicity couple to $H_{0}^{\prime}$. The full mass matrix is a direct product of several matrices. The neutrinos get their masses from

$$
M^{(\nu)}=\left(\begin{array}{cc}
m_{\mathrm{GUT}} f(r) e^{i n \theta} & m_{\mathrm{u}} h(r) e^{-i m \theta} \\
m_{\mathrm{u}} h(r) e^{-i m \theta} & 0
\end{array}\right),
$$

where $m_{\mathrm{u}}$ is the up quark mass and $m_{\mathrm{GUT}} \sim\left|\phi_{0}\right| \sim 10^{16} \mathrm{GeV}$. In the notation of (2) $\psi_{2}=\nu_{L}$ and $\psi_{1}=i \sigma^{2} \bar{\nu}_{R}^{T}$. The gauge eigenvalues of the two fields are 
then $q_{1}=n / 2$ and $q_{2}=-n / 2-m$. The other particles just couple to $H_{0}$ or $H_{0}^{\prime}$ [12].

At high temperatures only $\Phi_{126}$ is non-zero and $m_{\mathrm{u}}=0$, so only $\psi_{1}$ features in the zero mode analysis. Using (31) gives the number of neutrino zero modes as

$$
N_{L}=[n]_{+}, N_{R}=[-n]_{+} .
$$

At low temperatures $m_{\mathrm{u}} \neq 0$. This time

$$
N_{L}=[-2 m]_{+}, N_{R}=[2 m]_{+} .
$$

Since $|2 m|<|n|$, some of the zero modes will be destroyed. For a stable $n=1$ string all zero modes are destroyed. This is in agreement with results obtained in [8]. Thus, since higher $n$ strings almost certainly decay, there are zero modes before, but not after the electroweak phase transition. The neutral current in the string disperses 13 and any vortons formed would dissipate after about $10^{-10}$ sec [1]. Before the electroweak phase transition from about $10^{10} \mathrm{GeV}-10^{2} \mathrm{GeV}$ the universe would undergo a period of matter domination. Once the vortons dissipate there would be some reheating of the universe. However the electroweak interactions and physics below the phase transition would be unaffected.

In a more arbitrary $U(1) \times($ Standard Model $)$ theory, the ratio of $m$ and $n$ could be greater than 1 , in which case extra zero modes would be created at the electroweak phase transition.

The destruction of zero modes at phase transitions will occur in a wide range of models. In a theory with majorana mass terms, such as the right handed neutrino term above, the first version of the index theorem can be used (24). After a phase transition, if every fermion couples to a non-winding Higgs field, such as the electroweak Higgs field above, then all previously formed zero modes will be destroyed (see remarks after (24)).

\section{A Model with Persistent Zero Modes}

In this section we consider a model in which zero modes survive a subsequent symmetry breaking, despite coupling to a Higgs field with a non-winding component. Consider fermions which have the usual Yukawa couplings with two Higgs fields. Neglecting gauge fields, the form of the Lagrangian for each pair of fermions is then

$$
\mathcal{L}_{\mathrm{f}}=-i \bar{f}_{L} \gamma^{\mu} \partial_{\mu} f_{L}-i \bar{f}_{R} \gamma^{\mu} \partial_{\mu} f_{R}+m\left(\bar{f}_{L} \phi^{*} f_{R}+\bar{f}_{R} \phi f_{L}\right)
$$


where $\phi$ is a combination of the two Higgs fields, only one of which gives rise to a string. This leads to the Dirac equations

$$
\begin{gathered}
-i \gamma^{\mu} \partial_{\mu} f_{L}+m \phi^{*} f_{R}=0, \\
-i \gamma^{\mu} \partial_{\mu} f_{R}+m \phi f_{L}=0 .
\end{gathered}
$$

Using the following forms for the left and right-handed spinors,

$$
f_{L}=\left(\begin{array}{c}
A \\
B \\
-A \\
-B
\end{array}\right) \quad f_{R}=\left(\begin{array}{c}
C \\
D \\
C \\
D
\end{array}\right)
$$

the equations of motion become

$$
\left(\begin{array}{cccc}
w+k & i e^{-i \theta}\left(-\partial_{r}+\frac{i}{r} \partial_{\theta}\right) & m \phi^{*} & 0 \\
i e^{i \theta}\left(\partial_{r}+\frac{i}{r} \partial_{\theta}\right) & -w+k & 0 & m \phi^{*} \\
-m \phi & 0 & -w+k & i e^{-i \theta}\left(\partial_{r}-\frac{i}{r} \partial_{\theta}\right) \\
0 & -m \phi & i e^{i \theta}\left(-\partial_{r}-\frac{i}{r} \partial_{\theta}\right) & w+k
\end{array}\right)\left(\begin{array}{l}
A \\
B \\
C \\
D
\end{array}\right)=0
$$

We can now try the usual zero mode analysis [3]; let $w=k$ and set $A=D=0$. The equations of motion reduce to

$$
-i e^{-i \theta}\left(\partial_{r}-\frac{i}{r} \partial_{\theta}\right) B+m \phi^{*} C=0
$$

and

$$
-m \phi B-i e^{i \theta}\left(\partial_{r}+\frac{i}{r} \partial_{\theta}\right) C=0 .
$$

The Higgs field has two parts, a winding part from the string and a constant part from a second symmetry breaking,

$$
\phi=f(r) e^{i \theta}+p .
$$

Note that this form for $\phi$ cannot be written in the form used in (4) and so the index theorem developed in section 2 does not apply in this case. Changing variables to $X$ and $Y$ with

$$
X=\int_{0}^{r} f(\rho) d \rho+p r \cos \theta+c
$$


we have

$$
\left(\frac{\partial X}{\partial r} \pm \frac{i}{r} \frac{\partial X}{\partial \theta}\right)=f(r)+p\left[\cos \theta \pm \frac{i}{r}(-r \sin \theta)\right] .
$$

Taking $B$ and $C$ independent of $Y$ the equations of motion become

$$
-i e^{-i \theta}[f(r)+p(\cos \theta+i \sin \theta)] \partial_{X} B+m \phi^{*} C=0
$$

and

$$
-m \phi B-i e^{i \theta}[f(r)+p(\cos \theta-i \sin \theta)] \partial_{X} C=0 .
$$

The Higgs fields cancel and we have

$$
(-i B)_{, X}+m C=0 \quad, \quad m(-i B)+C_{, X}=0
$$

which have a solution,

$$
\left(\begin{array}{c}
(-i B) \\
C
\end{array}\right)=e^{-m X}\left(\begin{array}{l}
1 \\
1
\end{array}\right)
$$

We have explicitly constructed a zero mode after the second phase transition. The $X$ coordinate is similar to the usual radial coordinate, but 'centres' on the effective zero of the resultant Higgs field, rather than the core of the string.

\section{Index Theorems and Spectral Flow}

We have shown that zero modes can acquire masses at subsequent phase transitions. No matter how small this mass the spectrum of the Dirac operator changes significantly. If we compare the Dirac spectrum with a zero mode and a low lying bound state with infinitesimal mass (fig. 1), we see that an arbitrarily small perturbation to the zero mode introduces an entire new branch to the spectrum. Any massive state gives a spectrum that is symmetric about both the $w$ and $k$ axes, there is always a reference frame in which the particle is at rest and others where it is moving up or down the string. Conversely the zero mode, which is massless, can only move in one direction along the string and its spectrum is asymmetric. The transition from zero mode to low lying bound state causes drastic changes in the spectrum and can be brought about by infinitesimal changes in the value of one Higgs field. If we consider the species with the zero mode alone, this infinite susceptibility to the background fields appears unphysical. However, when we include the massless neutrino in the $S O(10)$ model the spectral changes 

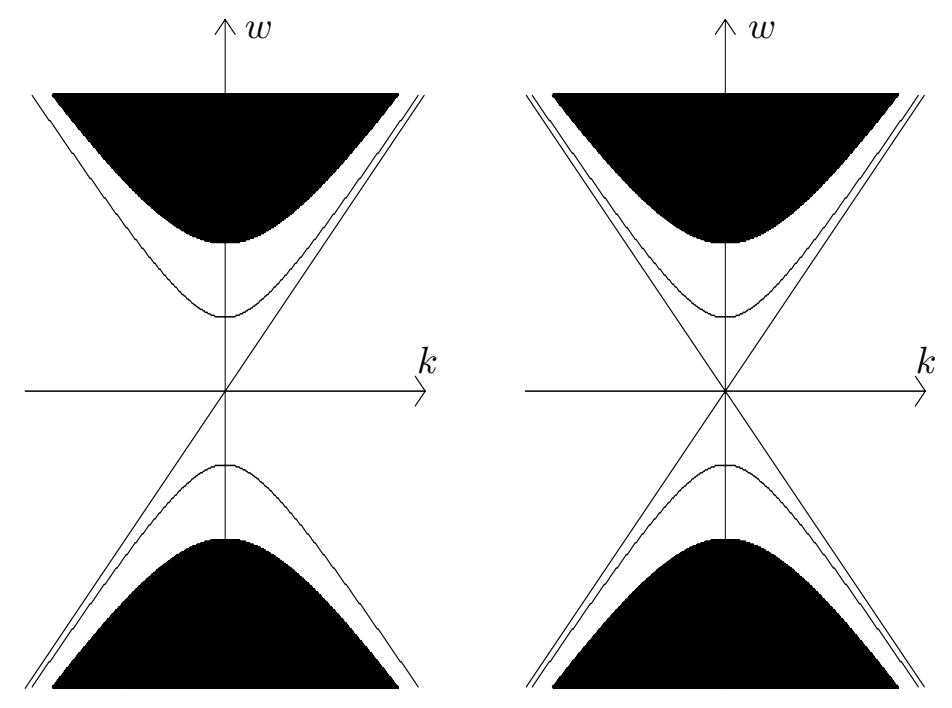

Figure 1: The Dirac spectrum with a zero mode (left) and a very low lying bound state (right). Both spectra also have a bound state and continuum.

are less worrying. For a small coupling between the two neutrinos, both the before and after spectra have a continuum of massless or nearly massless states. These states can be used to build the extra branch of the perturbed zero mode spectrum, allowing small changes in the overall spectrum for small changes in the background fields.

This observation leads us to conjecture that zero modes can be removed only if they become mixed with other states.

The coupling between the left and right handed neutrinos and the electroweak Higgs field need not be artificially small, the small mass of the light neutrino can be generated by the seesaw mechanism [14]. This coupling is present prior to the electroweak phase transition and allows transitions of the form $\nu_{L}+\bar{\nu}_{R} \rightarrow f \bar{f}$, where $f$ is any light fermion from the standard model and the intermediate state is an electroweak Higgs. Such interactions allow zero modes on the string to scatter from massless neutrinos in the surrounding plasma and provide a current damping mechanism that affects current build up prior to the electroweak transition. 


\section{Conclusions}

In this paper we have seen that the microphysics of cosmic strings can be influenced by subsequent phase transitions. Fermion zero modes, and consequently superconductivity, of the strings can be created or destroyed by such phase transitions. In determining whether or not a cosmic string is superconducting it is not enough to just consider this at formation, but to follow the microphysics through the multiple phase transitions that the system undergoes. It is possible for vortons formed at high energy to dissipate after a subsequent phase transition if the relevant fermion zero mode does not survive the phase transition, thus vortons bounds could be evaded. This is the case with $\nu_{R}$ zero modes on the $S O(10)$ string [8]. Prior to dissipation there could be a period of vorton domination, after the phase transition the universe would reheat and then evolve as normal.

To enable a systematic analysis of this effect we have derived a generalised index theorem. Our index theorem is especially applicable to theories where the fermions acquire mass from more than one Higgs field. We applied the index theorem and also considered spectral flow. As a result we conjecture that zero modes are destroyed when they mix with other fermions that acquire mass at a subsequent phase transition from a non-winding Higgs field.

\section{Acknowledgements}

This work was supported in part by PPARC, the EU under the HC programme (CHRX-CT94-0423) and Trinity College.

\section{References}

[1] M.B. Hindmarsh and T.W.B. Kibble, Rep. Prog. Phys. 58 (1995) 477; A. Vilenkin and E.P.S. Shellard, Cosmic Strings and Other Topological Defects (Cambridge University Press, 1994).

[2] W.B. Perkins and A.C. Davis, Nucl. Phys. B406 (1993) 377

[3] R. Jackiw and P. Rossi, Nucl. Phys. B190, (1981) 681.

[4] E. Witten, Nucl. Phys. B249 (1985) 557.

[5] R.L. Davis and E.P.S. Shellard, Nucl. Phys. B323 (1989) 209. 
[6] R. Brandenberger, B. Carter, A.C. Davis and M. Trodden, Phys. Rev. D54 (1996) 6059.

[7] A.C. Davis and W.B. Perkins, Phys. Lett. B390 (1997) 107.

[8] A.C. Davis and S.C. Davis, Phys. Rev. D 55 (1997) 1879.

[9] E.J. Weinberg, Phys. Rev. D 24 (1981) 2669.

[10] N. Ganoulis and G. Lazarides, Phys. Rev. D 38 (1988) 547.

[11] W.B. Perkins and A.C. Davis, Phys. Lett. B393 (1997) 46.

[12] A. Stern and U.A. Yajnik, Nucl. Phys. B267 (1986) 158.

[13] C.T. Hill and L.M. Widrow, Phys. Lett. B189 (1987) 17; M. Hindmarsh, Phys. Lett. B200 (1988) 429.

[14] M. Gell-Mann, P. Ramond and R. Slansky, in Supergravity, ed. D.Z.Freeman and P.van Nieuvenhuizen (North-Holland, 1979). 